\title{
DISCOVERY OF A GeV BLAZAR SHINING THROUGH THE GALACTIC PLANE
}

\author{
J. Vandenbroucke ${ }^{1}$, R. Buehler ${ }^{1}$, M. Ajello ${ }^{1}$, K. Bechtol $^{1}$, A. Bellini ${ }^{2,3}$, M. Bolte ${ }^{4}$, C. C. Cheung ${ }^{5,6}$, F. Civano $^{7}$, \\ D. Donato ${ }^{8}$, L. Fuhrmann ${ }^{9}$, S. FunK ${ }^{1}$, S. E. Healey ${ }^{1}$, A. B. Hill ${ }^{10,17}$, C. KNigge ${ }^{11}$, G. M. Madejski ${ }^{1}$, R. W. Romani ${ }^{1}$, \\ M. Santander-García ${ }^{12,13,14}$, M. S. Shaw ${ }^{1}$, D. Steeghs ${ }^{15}$, M. A. P. Torres ${ }^{7}$, A. Van EtTen ${ }^{1}$, and K. A. Williams ${ }^{16}$

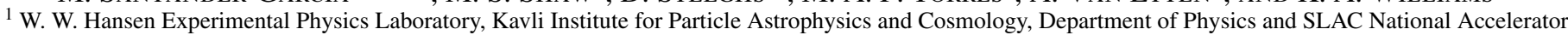 \\ Laboratory, Stanford University, Stanford, CA 94305, USA; justinv@ stanford.edu, buehler@stanford.edu \\ 2 Dipartimento di Astronomia, Università di Padova, I-35122 Padova, Italy \\ ${ }^{3}$ Space Telescope Science Institute, Baltimore, MD 21218, USA \\ ${ }^{4}$ Santa Cruz Institute for Particle Physics, Department of Physics and Department of Astronomy and Astrophysics, University of California at Santa Cruz, \\ Santa Cruz, CA 95064, USA \\ 5 Space Science Division, Naval Research Laboratory, Washington, DC 20375, USA \\ ${ }^{6}$ National Research Council Research Associate, National Academy of Sciences, Washington, DC 20001, USA \\ ${ }^{7}$ Harvard-Smithsonian Center for Astrophysics, Cambridge, MA 02138, USA \\ ${ }^{8}$ NASA Goddard Space Flight Center, Greenbelt, MD 20771, USA \\ ${ }^{9}$ Max-Planck-Institut für Radioastronomie, Auf dem Hügel 69, 53121 Bonn, Germany \\ ${ }^{10}$ Laboratoire d'Astrophysique de Grenoble (LAOG) UMR 5571, Université Joseph Fourier-Grenoble 1/CNRS, BP 53, 38041 Grenoble Cedex 09, France \\ ${ }^{11}$ School of Physics and Astronomy, University of Southampton, Highfield, Southampton, SO17 1BJ, UK \\ ${ }_{12}$ Instituto de Astrofísica de Canarias, E38205-La Laguna (Tenerife), Spain \\ ${ }^{13}$ Isaac Newton Group of Telescopes, E-38700 Sta. Cruz de la Palma, Spain \\ ${ }^{14}$ Departamento de Astrofisica, Universidad de La Laguna, E-38205 La Laguna, Tenerife, Spain \\ ${ }^{15}$ Department of Physics, University of Warwick, Coventry CV4 7AL, UK \\ ${ }^{16}$ Department of Astronomy, University of Texas, Austin, TX 75712, USA \\ Received 2010 April 6; accepted 2010 June 14; published 2010 July 14
}

\begin{abstract}
The Fermi Large Area Telescope (LAT) discovered a new gamma-ray source near the Galactic plane, Fermi J0109+6134, when it flared brightly in 2010 February. The low Galactic latitude $(b=-1.2)$ indicated that the source could be located within the Galaxy, which motivated rapid multi-wavelength follow-up including radio, optical, and X-ray observations. We report the results of analyzing all 19 months of LAT data for the source, and of X-ray observations with both Swift and the Chandra X-ray Observatory. We determined the source redshift, $z=0.783$, using a Keck Low-Resolution Imaging Spectrometer observation. Finally, we compiled a broadband spectral energy distribution (SED) from both historical and new observations contemporaneous with the 2010 February flare. The redshift, SED, optical line width, X-ray absorption, and multi-band variability indicate that this new $\mathrm{GeV}$ source is a blazar seen through the Galactic plane. Because several of the optical emission lines have equivalent width $>5 \AA$, this blazar belongs in the flat-spectrum radio quasar category.
\end{abstract}

Key words: galaxies: active

Online-only material: color figure

\section{INTRODUCTION}

Variability in gamma rays, as in other wavebands, is an essential diagnostic for identifying sources and determining emission mechanisms. Gamma-ray variability has been established for numerous extragalactic sources, all active galactic nuclei (AGNs) and gamma-ray bursts. Within the Galaxy, pulsars exhibit periodic variability on short (milliseconds to seconds) time scales corresponding to their spin period (and are otherwise relatively steady), and binary systems exhibit periodic variability on long (hours to days) time scales corresponding to their orbital period (Abdo et al. 2010a).

Accreting binary systems have exhibited transient outbursts during which their X-ray emission increases by a factor of up to $\sim 10^{6}$. One particular X-ray binary, Cygnus X-3 (a microquasar), has exhibited flaring activity in $\mathrm{GeV}$ gamma rays that is correlated with radio outbursts (Abdo et al. 2009b). Transient emission from Cygnus X-1 has also been claimed by AGILE (Sabatini et al. 2010b).

Flaring activity has not been established for Galactic $\mathrm{GeV}$ source classes other than binary systems. However, the Energetic

\footnotetext{
${ }^{17}$ Funded by contract ERC-StG-200911 from the European Community.
}

Gamma-Ray Experiment Telescope (EGRET) on board the Compton Gamma Ray Observatory did detect a bright gammaray transient near the Galactic plane, which had no radio-loud flat-spectrum radio counterpart and had properties inconsistent with those of an AGN or an isolated pulsar (Tavani et al. 1997). This source was never identified and has been interpreted as evidence for a new class of variable gamma-ray sources.

The unexplained EGRET event has motivated an ongoing search for new gamma-ray transients near the Galactic plane, as well as for variability among established Galactic gamma-ray sources. Prior to the discovery reported here, three transients near the Galactic plane (Hays \& Cheung 2009; Yasuda et al. 2009) have been detected with the Large Area Telescope (LAT) on board the Fermi Gamma-ray Space Telescope. None of them has yet been identified.

On 2010 February $1,{ }^{18}$ the LAT (Atwood et al. 2009) discovered a new, flaring source (Fermi J0109+6134) near the Galactic plane (Vandenbroucke \& Hill 2010). The AGILE gamma-ray telescope confirmed the Fermi LAT detection of the flare (Sabatini et al. 2010a). We report an analysis of the full 19 month LAT data set for the source region. We also report new

18 Dates and times are UTC throughout. 
multi-wavelength observations, including an optical spectrum from which we have determined the source redshift. Finally, we present a compilation of the broadband spectral energy distribution (SED) from both contemporaneous and historical data. The redshift, optical line width, SED shape, and multiband variability indicate a blazar identity for the source.

\section{FERMI LAT ANALYSIS}

We analyzed LAT data in a $15^{\circ}$ radius around Fermi J0109+6134 for the full data set acquired to date (2008 August 4 through 2010 February 23). We performed an unbinned likelihood fit to the data using the gtlike tool, which is part of the Fermi Science Tools. ${ }^{19}$ The statistical significance of the background-plus-source model relative to the background-only model is quantified with the "test statistic," $\mathrm{TS}=2 \Delta \log$ (likelihood). The unbinned likelihood method is described in Mattox et al. (1996) and Abdo et al. (2010a). The background model we used includes two diffuse components, one Galactic (model gll_iem_v02) and one isotropic (model isotropic_iem_v02), as well as nearby sources in the first Fermi LAT catalog (1FGL; Abdo et al. 2010a). The diffuse models, as well as the systematic uncertainties in source analysis associated with them, are described in Abdo et al. (2010a).

We fit Fermi J0109+6134 with a power-law energy spectrum, $\Phi(E)=\Phi_{0} E^{-\Gamma_{\gamma}}$, with both the normalization $\Phi_{0}$ and gammaray photon index $\Gamma_{\gamma}$ free. The normalization of each of the following background components was also free in the fit: each point source in 1FGL within a $6^{\circ}$ radius of Fermi J0109+6134, the nearby bright and variable binary LS I $+61^{\circ} 303$, the Galactic diffuse model, and the isotropic diffuse model. Only those events belonging to the highest quality (diffuse) class and with energies between $200 \mathrm{MeV}$ and $300 \mathrm{GeV}$ were included. Events with zenith angle greater than $105^{\circ}$ were removed to avoid contamination by gamma rays produced from cosmic-ray interactions in the atmosphere (Abdo et al. 2009a). We used the P6_V3_DIFFUSE instrument response functions.

No statistically significant flux was detected above background at the location of Fermi J0109+6134 in the 10 month LAT data set from 2008 August 4 through 2009 May 30: the TS for the source hypothesis using all data in this time interval is 3 . The $95 \%$ confidence upper limit on the flux of photons above $200 \mathrm{MeV}$ is $9.3 \times 10^{-9}$ photons $\mathrm{cm}^{-2} \mathrm{~s}^{-1}$.

For the subsequent nine month interval, from 2009 May 30 through 2010 February 23, Fermi J0109+6134 was detected with a TS of 639 , corresponding to a significance of $\sim 25 \sigma$. The integral flux in this interval is $(0.93 \pm 0.05) \times 10^{-7}$ photons $\mathrm{cm}^{-2} \mathrm{~s}^{-1}$ above $200 \mathrm{MeV}$, with $\Gamma_{\gamma}=2.59 \pm 0.06$. According to the best-fit source model, 1577 photons with energy greater than $200 \mathrm{MeV}$ are attributed to this source in the nine month time interval. From this analysis, the best-fit LAT position ${ }^{20}$ is (R.A. $=01^{\mathrm{h}} 09^{\mathrm{m}} 58^{\mathrm{s}} .4$, decl. $\left.=+61^{\circ} 32^{\prime} 58^{\prime \prime}\right)$. The LAT $68 \%$ error radius is $1^{\prime} .8$, corresponding to a $95 \%$ error radius of $3^{\prime} .0$.

No $\mathrm{GeV}$ source was previously known at this location. It was not detected in the EGR (Casandjian \& Grenier 2008), 3EG (Hartman et al. 1999), or 1FGL (Abdo et al. 2010a) catalog. The nearest source in 1FGL is 1FGL J0131.2+6121, an unassociated source 2.55 away (Abdo et al. 2010a).

The data from the nine month interval were divided into monthly time bins, and a likelihood analysis was performed in each. The source was detected significantly $(\mathrm{TS}>10)$ in each

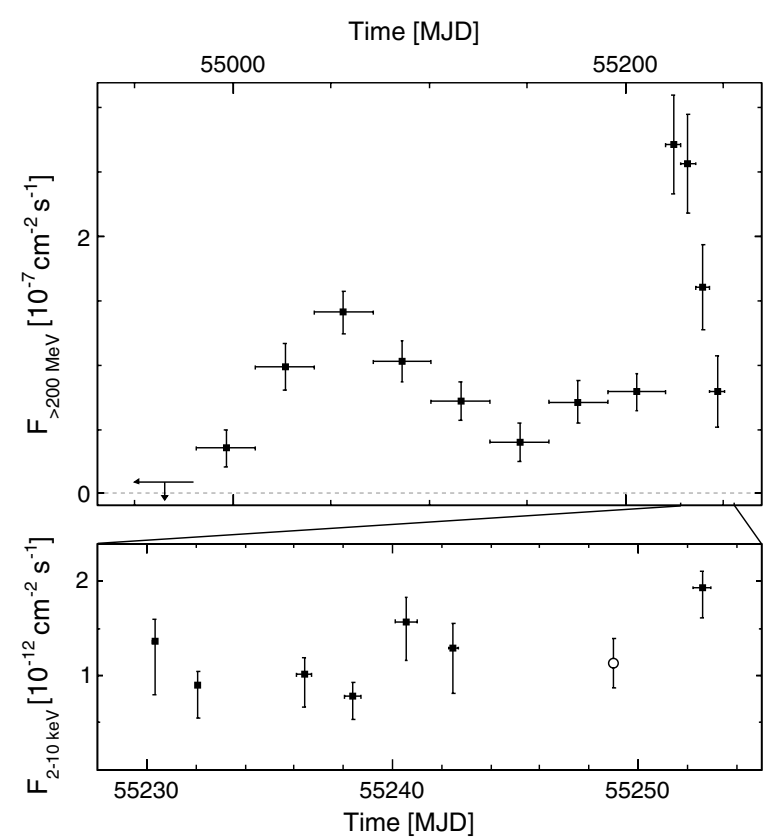

Figure 1. Upper panel: Fermi LAT light curve, for photons with energy above $200 \mathrm{MeV}$. The source was not detected between 2008 August 4 (MJD 54682) and 2009 May 30 (MJD 54971). This 10 month time interval is represented with a single upper limit. Lower panel: 2-10 keV X-ray light curve. There are seven Swift XRT observations (filled squares) and one Chandra observation (open circle). X-ray fluxes are shown as measured, without accounting for absorption.

month. The gamma-ray light curve is shown in Figure 1. The source is clearly variable on a $\sim 1$ month time scale, with two distinct flares peaking in 2009 August and 2010 February. The power-law index was determined for each time bin and showed no significant evidence of variation in the nine month analysis.

\section{COUNTERPARTS}

We have identified radio, optical, and X-ray counterparts of the new GeV gamma-ray source. Their positions are shown in Figure 2(a).

\subsection{Radio}

In archival radio data, we identified a likely flat-spectrum radio counterpart to the new GeV source: GT 0106+613 (VCS2 $\mathrm{J} 0109+6133)$. This is the only radio source known within the $68 \%$ LAT error radius. This source was first discovered by Gregory and Taylor in their radio survey of the northern Galactic plane (Taylor \& Gregory 1983; Gregory \& Taylor 1986), performed with the NRAO $91 \mathrm{~m}$ telescope at $5 \mathrm{GHz}$. They determined that it had compact morphology, in comparison with other sources that featured doublet or triplet morphology indicating one or two radio lobes in addition to a central object. A precise position for the radio source was later determined as part of the VCS2 very long baseline interferometry survey (Fomalont et al. 2003): (R.A. $=01^{\mathrm{h}} 09^{\mathrm{m}} 46^{\mathrm{s}} .34439 \pm 0.00013$, decl. $=+61^{\circ} 33^{\prime} 30^{\prime \prime} .4557 \pm 0^{\prime}$ '0003).

The high-resolution VCS2 image resolved few-milliarcsec (few-parsec, see redshift determination below) jet extension (Fomalont et al. 2003). This image ${ }^{21}$ is shown in Figure 2(b).

\footnotetext{
21 http://astrogeo.org/vcs2/vcs2_cat.html

\footnotetext{
19 Version v9r15p4.

20 We use the J2000.0 epoch throughout.
} 


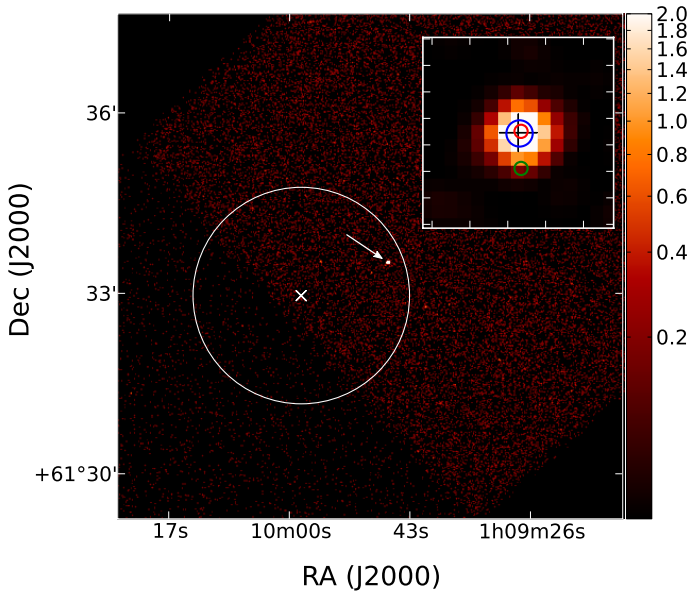

(a)

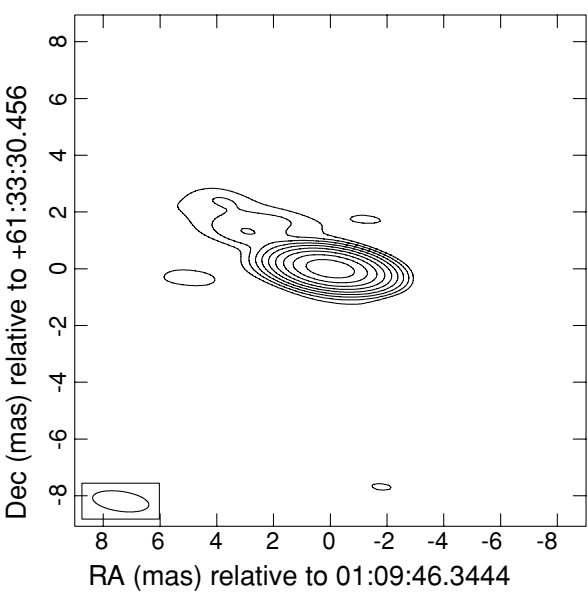

(b)

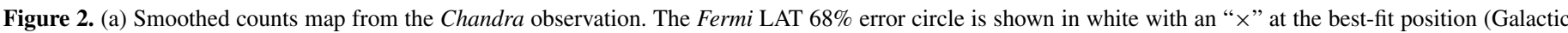

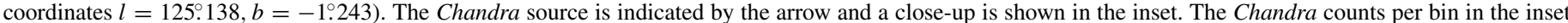

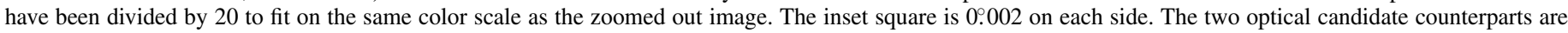

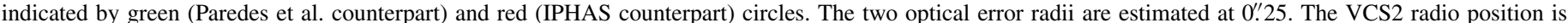

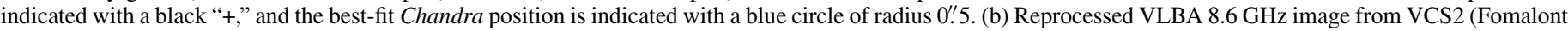

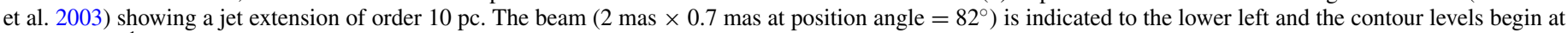
$1.5 \mathrm{mJy} \mathrm{bm}^{-1}$ and increase by factors of 2 .

(A color version of this figure is available in the online journal.)

\subsection{Optical}

Paredes et al. (1993) followed up the variable radio sources detected by Gregory and Taylor with optical observations. They detected a candidate optical counterpart of GT $0106+613$ with an $I$-band magnitude of 19.4 and a position (R.A. $=01^{\mathrm{h}} 09^{\mathrm{m}} 46.33$, decl. $=+61^{\circ} 33^{\prime} 29^{\prime \prime}$. 1) $1^{\prime \prime} .4$ offset from the VCS2 radio position. However, without a spectrum they were unable to determine whether the source was Galactic or extragalactic.

In addition to the Paredes et al. observations performed in 1992, the source lies within both the IPHAS (Drew et al. 2005) ${ }^{22}$ and UVEX (Groot et al. 2009) optical surveys of the northern Galactic plane. The IPHAS images (obtained between 2003 and 2006) show the Paredes et al. source, and they also show another optical source (IPHAS J010946.33+613330.5) which matches the radio position better than the Paredes et al. candidate and is $1^{\prime \prime} .4$ from the Paredes et al. candidate. This source has a position (R.A. $=01^{\mathrm{h}} 09^{\mathrm{m}} 46^{\mathrm{s}} 33$, decl. $\left.=+61^{\circ} 33^{\prime} 30^{\prime} .5\right)$ ) $0^{\prime} .1$ from the VCS2 radio position. The IPHAS and UVEX magnitudes are $\left(i^{\prime}=19.7, r^{\prime}-i^{\prime}=1.2\right)$ for the Paredes et al. counterpart and $\left(i^{\prime}=19.7, r^{\prime}-i^{\prime}=1.5\right)$ for the IPHAS counterpart that is closer to the radio position (Knigge et al. 2010).

\subsection{X-ray}

We obtained a series of seven observations of the source region with the Swift X-ray Telescope (XRT) ${ }^{23}$ between 2010 February 3 and 2010 February 25, each with a duration between 5 and $10 \mathrm{ks}$. We obtained a $10 \mathrm{ks}$ Chandra ACIS-S3 (AXAF CCD Imaging Spectrometer) observation, performed ${ }^{24}$ on 2010 February 21-22.

In the initial Swift XRT observation (Cheung et al. 2010), two X-ray sources were detected in the full XRT field. One was outside the LAT $95 \%$ error circle. The other was a bright source positionally coincident with the radio candidate, with (R.A. =

\footnotetext{
22 http://www.iphas.org

23 Swift Target ID 31604

24 Chandra Observation ID 11685, Sequence Number 702271
}

$01^{\mathrm{h}} 09^{\mathrm{m}} 46^{\mathrm{s}} .86$, decl. $=+61^{\circ} 33^{\prime} 29^{\prime} \cdot 3$ ) and a $90 \%$ error radius of 4". 4 from the initial $5 \mathrm{ks}$ observation.

Figure 2(a) shows the X-ray counts map obtained with the two Chandra chips that contain the Fermi $95 \%$ error circle. Two sources were detected. The first is a bright source within 1 "of the VCS2 radio position, with $503 \pm 25$ source photons. The second is a marginal source 3.9 away from the VCS2 position, well outside the LAT $95 \%$ error circle, with $18 \pm$ 6 source photons. We conclude that the first Chandra source is identified with the radio source with high confidence. The bestfit position of the Chandra source is (R.A. $=01^{\mathrm{h}} 09^{\mathrm{m}} 46^{\mathrm{s}} .338$, decl. $=+61^{\circ} 33^{\prime} 30^{\prime \prime}$.42).

While the small radio, optical, and X-ray error circles provide good confidence in the identification of these sources with one another, it remains to identify these sources unambiguously with the Fermi gamma-ray source, which has a much larger error circle. Using the gtsrcid tool (Abdo et al. 2010a), we estimate the chance probability of finding such a bright, flatspectrum radio source in the area of the Fermi error circle to be $4 \times 10^{-5}$.

The Galactic column density in this direction is estimated to be $N_{\mathrm{H}}=5.5 \times 10^{21} \mathrm{~cm}^{-2}$ from the Leiden/Argentine/ Bonn (LAB) $21 \mathrm{~cm}$ survey (Kalberla et al. 2005). We fit the Chandra X-ray spectrum with a hard $\left(\Gamma_{X}=1.19_{-0.15}^{+0.16}\right)$ absorbed $\left(N_{\mathrm{H}}=9.2_{-1.3}^{+1.5} \times 10^{21} \mathrm{~cm}^{-2}\right)$ power law, with $\chi^{2}=40.3$ for $61-3=58$ degrees of freedom. The photoelectric absorption model uses cross sections from Morrison \& McCammon (1983) and relative abundances from Anders \& Ebihara (1982). It is not surprising that the column density estimated from the X-ray absorption is significantly larger than that estimated from the LAB radio survey data, particularly for this direction within $10^{\circ}$ of the Galactic plane. The X-ray estimate is likely more reliable than the LAB estimate due to uncertainty in the neutral hydrogen spin temperature, which is used to infer column density from the $21 \mathrm{~cm}$ data.

The measured (absorbed) Chandra flux is $1.15 \times 10^{-12} \mathrm{erg}$ $\mathrm{cm}^{-2} \mathrm{~s}^{-1}$ in the $2-10 \mathrm{keV}$ band. The model (unabsorbed) flux is $1.23 \times 10^{-12} \mathrm{erg} \mathrm{cm}^{-2} \mathrm{~s}^{-1}$ in the same band. The absolute 


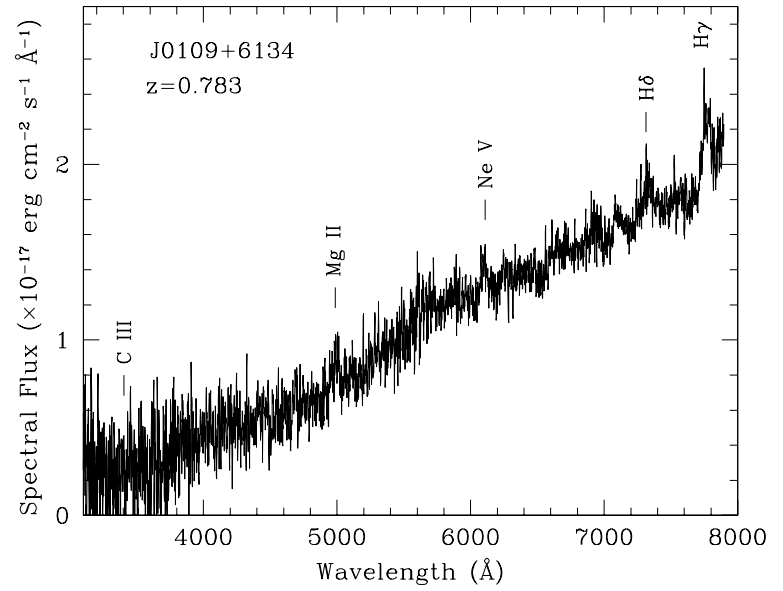

Figure 3. Optical spectrum obtained with Keck. Extinction has not been corrected.

flux uncertainty is $\sim 25 \%$. We also fit the spectrum including a thermal component (a $k T=1.5 \mathrm{keV}$ MEKAL spectrum) fixed at the newly determined (see Section 4) redshift, $z=0.783$.

We analyzed Swift Burst Alert Telescope (BAT) data from 2004-2009 and found that the source was detected with a significance of $\sim 4.5 \sigma$. We used this data to estimate the spectrum in the 14-195 keV band, following the procedure described in Ajello et al. (2008, 2009). The flux in this band is $1.03_{-0.39}^{+0.41} \times 10^{-11} \mathrm{erg} \mathrm{cm}^{-2} \mathrm{~s}^{-1}$.

\section{OPTICAL SPECTRUM AND REDSHIFT}

We obtained an optical spectrum on 2010 February 9 using the Low-Resolution Imaging Spectrometer (LRIS) with the atmospheric dispersion corrector (Oke et al. 1995) at the Keck 1 telescope. A $1^{\prime \prime}$ longslit was placed at a position angle of 12.5 east of north in order to include both optical sources in a single pointing. The observations were performed at high airmass and as a consequence the image quality full-width half-maximum was $\sim 1^{\prime \prime} .2$.

Data reduction was performed with the IRAF package using standard techniques: an optimal extraction algorithm to maximize the signal-to-noise ratio $(\mathrm{S} / \mathrm{N})$; wavelength calibration from sky lines; spectrophotometric calibration and telluric subtraction from observations of G191-B2B; and visual inspection to clean cosmic rays. Multiple exposures were combined into a single spectrum, weighted by the $\mathrm{S} / \mathrm{N}$.

The Paredes et al. (1993) candidate showed Balmer absorption at rest, indicating that it is a field star. The IPHAS counterpart showed a red continuum-dominated spectrum, with broad UV emission lines consistent with a redshifted blazar subject to Galactic extinction.

The spectrum of the IPHAS counterpart is shown in Figure 3. Five emission lines were identified: $\mathrm{C}$ III, $\mathrm{Mg}$ II, $\mathrm{Ne} \mathrm{v}, \mathrm{H} \delta$, and $\mathrm{H} \gamma$. From the spectrum we determine the redshift of this source to be 0.783 . The equivalent width of each of the $\mathrm{Mg}$ II, $\mathrm{Ne} \mathrm{v}$, $\mathrm{H} \delta$, and $\mathrm{H} \gamma$ lines exceeds $5 \AA$. The $\mathrm{S} / \mathrm{N}$ of the $\mathrm{C}$ III line is too low to estimate its width. Because the strongest emission line width was greater than $5 \AA$, we classify this blazar as a flat-spectrum radio quasar rather than a BL Lacertae, following the classification scheme of the Candidate Gamma-Ray Blazar Survey (Healey et al. 2008) and the first Fermi LAT AGN catalog (Abdo et al. 2010b).

A fit to the continuum in the Keck spectrum (between $380 \mathrm{~nm}$ and $800 \mathrm{~nm}$ ) gives $v F_{v}=8.3_{-3.2}^{+3.6} \times 10^{-13} \mathrm{erg} \mathrm{cm}^{-2} \mathrm{~s}^{-1}$ at
$5.45 \times 10^{14} \mathrm{~Hz}(550 \mathrm{~nm})$ and $\alpha-1=0.1_{-0.4}^{+0.3}$, where $v F_{v} \propto$ $v^{-(\alpha-1)}$ and the optical photon index is $\Gamma_{\mathrm{opt}}=\alpha+1$. Galactic extinction has been removed using the maps of Schlegel et al. (1998). The uncertainty on the normalization and index include $10 \%$ uncertainty in the extinction maps and $30 \%$ uncertainty in the absolute spectrophotometry due to slit loss and nearby source confusion.

\section{RADIO, OPTICAL, AND X-RAY VARIABILITY}

Taylor \& Gregory (1983) determined that this radio source exhibited long-term $(\geqslant 1 \mathrm{yr})$ and possibly short-term $(<1 \mathrm{yr})$ variability. Only a few percent of the sources in their northern Galactic plane survey exhibited variability, and they requested additional observations to determine which if any were Galactic. Duric \& Gregory (1988) established that the source exhibited short-term as well as long-term radio variability.

In addition to historical (pre-2010) radio flux densities measured at several frequencies between 0.365 and $22 \mathrm{GHz}$, a new radio spectrum, contemporaneous with the 2010 February flare, was measured with the Effelsberg $100 \mathrm{~m}$ telescope on 2010 February 4. The flux density was measured at several frequencies between 2.6 and $32 \mathrm{GHz}$. At 8 (22) GHz, the flux density was greater than archival values by $\sim 20 \%(\sim 50 \%)$ (Fuhrmann et al. 2010), indicating radio flaring behavior contemporaneous with the gamma-ray flare.

There is marginal evidence for optical variability over the several years of IPHAS frames. The absence of the IPHAS source in the Paredes et al. (1993) observations (when the Paredes et al. counterpart was detected clearly) provides stronger evidence of optical variability. Both candidate counterparts were imaged using a $120 \mathrm{~s}$ exposure of the ACAM camera on the William Herschel Telescope on 2010 February 5 to check the brightness at the time of the observed gamma-ray flare. The Paredes et al. candidate had $r^{\prime}=20.9$ and the IPHAS candidate had $r^{\prime}=21.2$, each the same as determined from the 2003-2006 IPHAS images (Knigge et al. 2010).

Figure 1 shows the X-ray light curve compiled from the Swift and Chandra observations. In the 2010 February 3-25 time interval, the X-ray flux shows no significant variability.

\section{SPECTRAL ENERGY DISTRIBUTION AND INTERPRETATION}

We compiled historical and contemporaneous multi-band spectra in the SED shown in Figure 4. The two-bump shape is consistent with typical blazar SEDs where the low-energy bump is interpreted as synchrotron emission and the high-energy bump is interpreted as inverse Compton emission.

In other blazars, it is not unusual to observe variability in the gamma-ray band without simultaneous correlated variability in the X-ray band (Fermi-Lat Collaboration et al. 2010). Although both the X-ray and the gamma-ray photons are believed to be produced by inverse Compton emission, they are produced from populations of electrons that have different energies and could be located in different regions.

For reference, we fit a parameterization (third degree polynomial) to each bump, following the method of Abdo et al. (2010c). This fit gives a synchrotron peak at $\sim 10^{13} \mathrm{~Hz}$ and a Compton peak at $\sim 10^{22} \mathrm{~Hz}$, making this a Low Synchrotron Peaked blazar following the classification scheme of Abdo et al. (2010c).

The redshift, SED shape, optical line width, and multi-band variability of this source indicate that it is a background blazar 


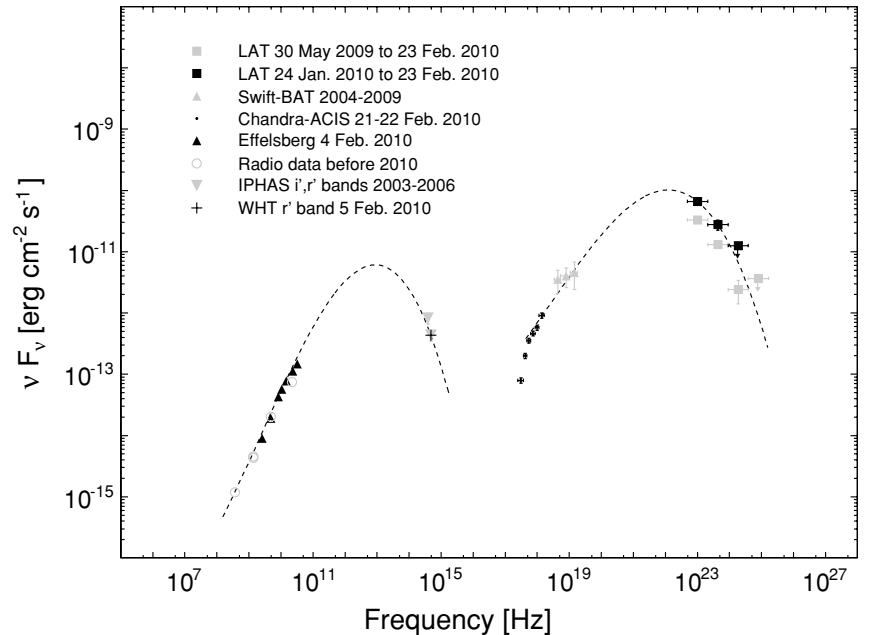

Figure 4. Spectral energy distribution. Historical data are shown in gray. Simultaneous data acquired during the 2010 February flare are shown in black. Extinction has been removed from the optical points and not from the X-ray points. For Fermi energy bins with no statistically significant flux detected (TS < 9), a 95\% upper limit was calculated and is indicated with arrows. Historical radio data are from Douglas et al. (1996), White \& Becker (1992), Condon et al. (1998), Becker et al. (1991), and Petrov et al. (2007). Dashed lines indicate a third-order polynomial fit to each bump.

seen shining through the Galactic plane. It provides an example of extragalactic $\mathrm{GeV}$ source identification and characterization in the difficult region very close to the Galactic plane. Blazar catalogs generally avoid the region within $\pm 10^{\circ}$ of the Galactic plane due to the significant extinction and source confusion in this region.

X-ray measurements of background blazars can improve our understanding of the Galactic interstellar medium. With improved statistics, the absorption fit to the X-ray spectrum may constrain both the column density and composition in the direction of this source, independent of the LAB $21 \mathrm{~cm}$ survey. Blazars are believed to have negligible intrinsic absorption (Perlman et al. 2005).

This source provides the first example of successfully determining the identity of a transient $\mathrm{GeV}$ source discovered near the Galactic plane by following up with collaborative multiobservatory, multi-wavelength observations. Monitoring of this blazar is ongoing with the Fermi LAT and other facilities. The three other Galactic plane transients detected with the Fermi LAT in its first 19 months of operation remain unidentified. Observation and analysis are ongoing to identify these transients and to detect new GeV transients near the Galactic plane. With its large field of view and frequent full-sky scans, Fermi is very well suited to discover more $\mathrm{GeV}$ transients across the sky and in particular in the Galactic plane. Fermi observations of these transients will likely lead to new understanding of known $\mathrm{GeV}$ source classes as well as the discovery of new classes of $\mathrm{GeV}$ emitters.

We thank the Chandra and Swift teams for timely and high-quality X-ray observations. We are grateful for valuable comments on the manuscript from Berrie Giebels. J.V. is supported by a Kavli Fellowship from the Kavli Foundation. The Fermi LAT Collaboration acknowledges support from a number of agencies and institutes for both the development and the operation of the LAT as well as scientific data analysis. These include NASA and DOE in the United States, CEA/Irfu and IN2P3/CNRS in France, ASI and INFN in Italy, MEXT, KEK, and JAXA in Japan, and the K. A. Wallenberg Foundation, the Swedish Research Council, and the National Space Board in Sweden. Additional support from INAF in Italy and CNES in France for science analysis during the operations phase is also gratefully acknowledged.

Facilities: Fermi (LAT), CXO (ACIS), Keck: Interferometer (LRIS), Swift (XRT, BAT), Effelsberg, ING:Herschel.

\section{REFERENCES}

Abdo, A. A., et al. 2009a, Phys. Rev. D, 80, 122004

Abdo, A. A., et al. 2009b, Science, 326, 1512

Abdo, A. A., et al. 2010a, ApJS, 188, 405

Abdo, A. A., et al. 2010b, ApJ, 715, 429

Abdo, A. A., et al. 2010c, ApJ, 716, 30

Ajello, M., et al. 2008, ApJ, 673, 96

Ajello, M., et al. 2009, ApJ, 699, 603

Anders, E., \& Ebihara, M. 1982, Geochim. Cosmochim. Acta, 46, 2363

Atwood, W. B., et al. 2009, ApJ, 697, 1071

Becker, R. H., White, R. L., \& Edwards, A. L. 1991, ApJS, 75, 1

Casandjian, J., \& Grenier, I. A. 2008, A\&A, 489, 849

Cheung, C. C., Donato, D., Ajello, M., Buehler, R., \& Vandenbroucke, J. 2010, ATel, 2420, 1

Condon, J. J., Cotton, W. D., Greisen, E. W., Yin, Q. F., Perley, R. A., Taylor, G. B., \& Broderick, J. J. 1998, AJ, 115, 1693

Douglas, J. N., Bash, F. N., Bozyan, F. A., Torrence, G. W., \& Wolfe, C. 1996, AJ, 111,1945

Drew, J. E., et al. 2005, MNRAS, 362, 753

Duric, N., \& Gregory, P. C. 1988, AJ, 95, 1149

Fermi-LAT Collaboration, et al. 2010, Nature, 463, 919

Fomalont, E. B., Petrov, L., MacMillan, D. S., Gordon, D., \& Ma, C. 2003, AJ, 126,2562

Fuhrmann, L., Bach, U., Nestoras, I., Krichbaum, T. P., \& Angelakis, E. 2010, ATel, 2428, 1

Gregory, P. C., \& Taylor, A. R. 1986, AJ, 92, 371

Groot, P. J., et al. 2009, MNRAS, 399, 323

Hartman, R. C., et al. 1999, ApJS, 123, 79

Hays, E., \& Cheung, C. C. 2009, Proc. 31st ICRC, (Lodz), http://icrc2009.uni. lodz.pl/proc/pdf/icrc0818.pdf

Healey, S. E., et al. 2008, ApJS, 175, 97

Kalberla, P. M. W., Burton, W. B., Hartmann, D., Arnal, E. M., Bajaja, E., Morras, R., \& Pöppel, W. G. L. 2005, A\&A, 440, 775

Knigge, C., et al. 2010, ATel, 2429, 1

Mattox, J. R., et al. 1996, ApJ, 461, 396

Morrison, R., \& McCammon, D. 1983, ApJ, 270, 119

Oke, J. B., et al. 1995, PASP, 107, 375

Paredes, J. M., Marti, J., Jordi, C., Trullols, E., \& Peracaula, M. 1993, A\&AS, 102,381

Perlman, E. S., et al. 2005, ApJ, 625, 727

Petrov, L., Hirota, T., Honma, M., Shibata, K. M., Jike, T., \& Kobayashi, H. 2007, AJ, 133, 2487

Sabatini, S., et al. 2010a, ATel, 2416, 1

Sabatini, S., et al. 2010b, ApJ, 712, L10

Schlegel, D. J., Finkbeiner, D. P., \& Davis, M. 1998, ApJ, 500, 525

Tavani, M., et al. 1997, ApJ, 479, L109

Taylor, A. R., \& Gregory, P. C. 1983, AJ, 88, 1784

Vandenbroucke, J., \& Hill, A. B. 2010, ATel, 2414, 1

White, R. L., \& Becker, R. H. 1992, ApJS, 79, 331

Yasuda, H., Takahashi, H., \& McConville, W. 2009, ATel, 2081, 1 